\title{
Catechol-O-methyltransferase genotype is associated with plasma total homocysteine levels and may increase venous thrombosis risk
}

\author{
Henkjan Gellekink',2*, Jan-Willem Muntjewerff4*, Sita H. H. M. Vermeulen², Ad R. M. M. Hermus², Henk J. Blom', \\ Martin den Heijer 2,3 \\ 'Laboratory of Pediatrics and Neurology, ${ }^{2}$ Department of Endocrinology and ${ }^{3}$ Department of Epidemiology and Biostatistics, Radboud \\ University Nijmegen Medical Centre, Nijmegen, The Netherlands; ${ }^{4}$ GGz Nijmegen, Mental Health Institute, Nijmegen, The Netherlands
}

\begin{abstract}
Summary
A disturbed methylation has been proposed as a mechanism via which homocysteine is associated with diseases like vascular disease, neural tube defects and mental disorders. CatecholO-methyltransferase (COMT) is involved in the S-adenosylmethionine-dependent methylation of catecholamines and catecholestrogens and in this way contributes to homocysteine synthesis. COMT dysfunction has been related to schizophrenia and breast cancer. We hypothesized that COMT dysfunction by virtue of functional genetic polymorphisms may affect plasma total homocysteine (tHcy). Our primary objective was to study the association between common COMT polymorphisms and thcy. Secondly, we evaluated these polymorphisms as a risk factor for recurrent venous thrombosis. We obtained genotype data from four polymorphisms in the COMT gene (rs2097603,
\end{abstract}

\section{Keywords}

Homocysteine, haplotype, catechol-O-methyltransferase, venous thrombosis

\section{Introduction}

A disturbed homocysteine metabolism has been associated with diseases of the vascular system, both of arterial and venous origin (1-3). In addition, high plasma total homocysteine (tHcy) increases the risk of spina bifida, and mental disorders like schizophrenia and Alzheimer's disease (4-6). The mechanism of how homocysteine is related to disease is still obscure (7), but there are strong indications that a disturbed transmethylation may partly explain this association (8-11). Several studies show that plasma total homocysteine levels (tHcy) correlate well with plasma S-adenosylhomocysteine (AdoHcy) $(12,13)$, a strong inhibitor of S-adenosylmethionine (AdoMet)-dependent methyl- rs4633, rs4680 [324G>A] and rs I74699) from 40I populationbased controls. We performed haplotype analysis to investigate the association between common haplotypes and tHcy. In addition, we assessed the rs 4680 variant as a genetic risk factor in a case-control study on recurrent venous thrombosis $(n=169)$. We identified a common haplotype that was significantly associated with tHcy levels. This effect was largely explained by the rs 4680 variant, resulting in an increase in tHcy of $10.4 \%(95 \% \mathrm{Cl}$ 0.01 to $0.21, p=0.03$ ) for $324 A A$ compared with $324 G G$ subjects. Interestingly, we found that the 324AA genotype was more common in venous thrombosis patients (OR I.6I [ $95 \% \mathrm{Cl} 0.97$ to 2.65], $p=0.06$ ) compared to control subjects. We show that the COMT rs 4680 variant modulates tHcy, and might be associated with venous thrombosis risk as well. ation. Given the importance of methylation of nucleic acids, proteins, lipids $(8,10,14,15)$, but also hormones and neurotransmitters $(16,17)$, it seems plausible that the inhibition or dysfunction of specific methyltransferases affect critical processes and hence confer a higher risk of disease.

The enzyme Catechol-O-methyltransferase (COMT, E.C. 2.1.1.6) is one of the methyltransferases that is highly susceptible to inhibition by AdoHcy (18). COMT represents a major pathway in the degradation of catecholamine neurotransmitters, like dopamine and (nor)adrenaline, and catecholestrogens. Hence, COMT dysfunction has been implicated in complex diseases like schizophrenia, Parkinson's disease and breast cancer $(17,19-21)$. 
COMT enzyme exists as a membrane-bound (MB) and soluble (S) isoform, the expression of which is regulated by two different promoters. A common 324G $>$ A polymorphism (rs4680) in the COMT gene, resulting in a valine-to-methionine substitution at position 108 (S-isoform) and 158 (MB-isoform), has been studied extensively for its effect on enzyme activity and expression, although the data is non-consistent $(22,23)$.

The metabolic pathways of COMT and homocysteine are interconnected as the O-methylation of catecholamines and catecholestrogens catalyzed by COMT produces homocysteine (Fig. 1). Therefore, functional variants within the COMT gene may influence tHcy levels and possibly reflect disturbed transmethylation capacity implicated in COMT-related diseases. This is illustrated by the fact that we did find an interaction between MTHFR and COMT in relation to schizophrenia risk (25).

The primary objective of our study was to investigate the effect of genetic variations within the COMT gene on tHcy by means of haplotype analysis. Considering the controversy whether the $324 \mathrm{G}>\mathrm{A}$ variant is causally related to decreased enzyme activity or expression, we included three other variants (rs2097603) [22], rs4633 and rs 174699) in or directly adjacent to the COMT gene in our analyses. Secondly we evaluated thesepolymorphisms as a risk factor for recurrent venous thrombosis.

\section{Material and methods}

\section{Subjects used in the present study}

The study group was recruited via a general practice in The Hague, the Netherlands, and has been described in more detail elsewhere (25). Recurrent venous thrombosis cases were selected from the files of the anticoagulant clinic in Leyenburg Hospital in The Hague, The Netherlands, and control subjects were recruited via a general practice; pregnancy was the only exclusion criterion. For the current association study, DNA was available from 438 control subjects and 169 recurrent venous thrombosis patients. With these numbers we had power of 50 to $78 \%$ to detect a difference of 10 to $15 \%$ in tHcy (two sided $\alpha=0.05$ ). The power to detect an effect of COMT genotype on venous thrombosis was $54 \%$.

\section{Biochemical parameters}

Blood samples were drawn after an overnight fast from the antecubital vein in $5 \mathrm{ml}$ Vacutainer tubes and $4.5 \mathrm{ml}$ EDTA vacuum glass tubes for determination of total plasma homocysteine and for DNA extraction. EDTA samples for homocysteine measurement were placed on ice immediately and centrifuged at 3,500 g for 5 minutes ( $\mathrm{min}$ ) with minimal delay. The plasma was separated and then stored at $-20^{\circ} \mathrm{C}$. Total plasma homocysteine concentrations were measured by an automated high-performance liquid chromatography method with reverse phase separation and fluorescent detection (Gilson 232-401 sample processor, Spectra Physics 8800 solvent delivery system and LC 304 fluorometer) (26). DNA extraction was performed as described previously (27), and the DNA was stored at $4^{\circ} \mathrm{C}$.

\section{Genotype analysis}

We genotyped four single nucleotide polymorphisms (SNPs) distributed over the gene of interest: rs2097603, rs4633, rs4680 (sometimes referred to as rs165688) and rs174699. The SNPs were chosen based on frequency, functionality and location within the gene. All four created or abolished an enzyme restriction site allowing a simple screening based on restriction-fragment length polymorphism (RFLP) analysis. Screening conditions were similar in all analyses. For details see Table 1. PCR conditions: $4 \mathrm{~min}$ at $94^{\circ} \mathrm{C}, 35$ cycles of $94^{\circ} \mathrm{C} / 60$ seconds (s), $52-60^{\circ} \mathrm{C} / 60 \mathrm{~s}$, and $72^{\circ} \mathrm{C} / 30 \mathrm{~s}$, and a final extension of $7 \mathrm{~min}$ at $72^{\circ} \mathrm{C}$. Each PCR reaction mixture contained $50 \mathrm{ng}$ of both forward and reverse primer (Biolegio BV, The Netherlands), $200 \mu \mathrm{M}$ dNTPs, $10 \mathrm{mM}$ Tris-HCl buffer (pH 8.2), $50 \mathrm{mM} \mathrm{KCl}$, 2.0-4.0 $\mathrm{mM} \mathrm{MgCl}_{2}, 0.5 \mathrm{U}$ of recombinant Taq polymerase, $5 \%$ DMSO (Invitrogen, The Netherlands) and 75 ng DNA. The resulting PCR product was digested by at least 10 units of restriction enzyme (all from New England Biolabs, Inc.) and incubated for 3 hours or overnight at $37^{\circ} \mathrm{C}$. The digests were analyzed by gel electrophoresis on a $3 \%$ or $4 \%$ agarose gel, stained with ethidiumbromide and visualized by UV. In all PCR amplifications and restriction analyses, DNA samples from which the genotype had been identified by sequence analysis served as positive controls. From 401 population-based controls, genotype data was available for all four SNP's (although the total number of genotyped individuals were different for each polymorphism).
Figure I: Simplified representation of homocysteine metabolism and the location of Catechol-o-methyltransferase (COMT) enzyme within this metabolism. Abbreviations: Met, methionine; AdoMet, S-adenosylmethionine; AdoHcy, S-adenosylhomocysteine; Hcy, homocysteine; MS, Methionine Synthase; THF, tetrahydrofolate; MTHFR, methylenetetrahydrofolate reductase.

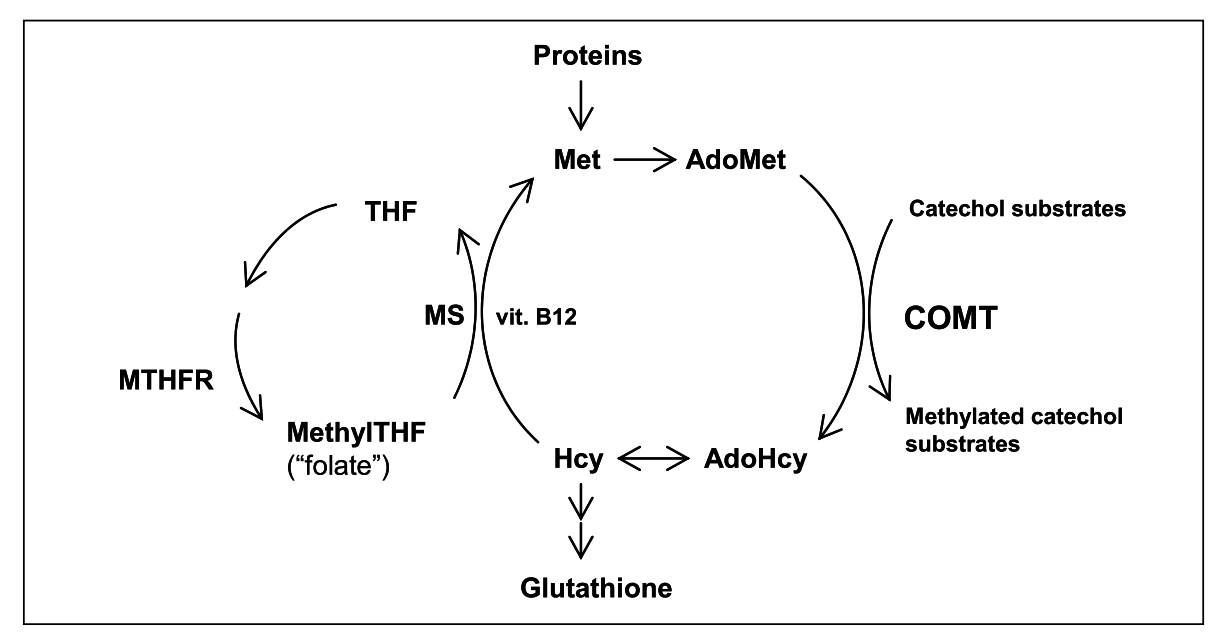




\begin{tabular}{|c|c|c|c|}
\hline Variable & $\begin{array}{l}\text { Cases } \\
(\mathrm{n}=185)\end{array}$ & $\begin{array}{l}\text { Controls } \\
(n=500)\end{array}$ & P-value \\
\hline Age (year) & $62(42-79)$ & $50(34-69)$ & $<0.01$ \\
\hline Sex (men) & $94(51 \%)$ & $208(42 \%)$ & 0.03 \\
\hline Post-menopausal women & $64(35 \%)$ & $138(28 \%)$ & $<0.01$ \\
\hline Time between Ist event and study (years) & 17 (range I to 58) & - & - \\
\hline Time between last event and study (years) & 7 (range I to 30) & - & - \\
\hline $\begin{array}{l}\text { Type of venous thrombosis } \\
\text { - Only pulmonary embolism (PE) } \\
\text { - Only deep vein thrombosis (DVT) } \\
\text { - Both DVT and PE }\end{array}$ & $\begin{array}{l}43 \\
73 \\
69\end{array}$ & - & - \\
\hline Smoking (yes/no) & $61(33 \%)$ & $167(34 \%)$ & 0.9 \\
\hline B-vitamin (yes/no) & $23(12 \%)$ & $76(15 \%)$ & 0.9 \\
\hline Homocysteine $(\mu \mathrm{M})$ & $12.2(8.3-20.8)$ & $10.7(6.7-15.5)$ & $<0.01$ \\
\hline Creatinine $(\mu \mathrm{M})$ & $81(6|-| \mid 2)$ & $74(55-99)$ & $<0.01$ \\
\hline Folate (nM) & I $3.5(7.7-23.5)$ & $12.7(7.0-23.6)$ & 0.2 \\
\hline Vitamin $B 12(\mu \mathrm{M})$ & $24 I(118-466)$ & $217(125-389)$ & 0.04 \\
\hline Vitamin B6 (nM) & $25.8(12.7-55.7)$ & $27.6(15.7-54.8)$ & 0.03 \\
\hline Vitamin B2 (nM) & $10.2(4.9-37.1)$ & $9.1(4.0-25.9)$ & 0.07 \\
\hline Factor $V$ Leiden $(\mathrm{MAF})^{\dagger}$ & $14.6 \%$ & $3.3 \%$ & $<0.01$ \\
\hline Prothrombin G20210A (MAF) ${ }^{\dagger}$ & $2.9 \%$ & $1.6 \%$ & 0.12 \\
\hline MTHFR C677T (MAF) $)^{\dagger}$ & $33.3 \%$ & $29.1 \%$ & 0.21 \\
\hline
\end{tabular}

Table I: Baseline characteristics of patients with recurrent venous thrombosis and control subjects*.

Table 2: Relevant data of analyzed polymorphisms and method of screening.

\begin{tabular}{|c|c|c|c|c|c|c|c|c|}
\hline Variant & $\begin{array}{l}\text { Reported } \\
\text { MAF }^{\mathrm{a}}\end{array}$ & DNA & Protein $^{b}$ & $\begin{array}{l}\text { HW P- } \\
\text { value }\end{array}$ & Primers (5'à 3') & $\begin{array}{l}\text { Validation } \\
\text { method }\end{array}$ & $\begin{array}{l}{\left[\mathrm{Mg}^{2+}\right]} \\
(\mathrm{mM})\end{array}$ & $\begin{array}{l}\mathbf{T}_{\text {ann }} \\
\left({ }^{\circ} \mathbf{C}\right)\end{array}$ \\
\hline rs2097603 & $0.39(\mathrm{G})$ & $\begin{array}{l}\text { 5' region } A>G \\
\text { ( } P 2 \text { promoter) }\end{array}$ & - & 0.87 & $\begin{array}{l}\mathrm{F} \text { aatttggctattgccgtgtc } \\
\mathrm{R} \text { gtcccataaaaggggattcg }\end{array}$ & + HindIII & 2.0 & 52 \\
\hline rs4633 & $0.39(\mathrm{~T})$ & $36 \mathrm{~T}>\mathrm{C}$ & $\mathrm{HI} 2 \mathrm{H}$ & 0.52 & $\begin{array}{l}\text { F acaacctgctcatgggtgac } \\
R \text { tcctgtaagggctttgatgc }\end{array}$ & + Pmll & 4.0 & 60 \\
\hline rs4680 & $0.36-0.52(\mathrm{~A})$ & $324 G>A$ & VI08M & 0.40 & $\begin{array}{l}\mathrm{F} \text { tactgtggctactcagctgtgc } \\
\mathrm{R} \text { gtgaacgtggtgtgaacacc }\end{array}$ & + Nlalll & 2.0 & 59 \\
\hline
\end{tabular}

In addition, data of four other reported variants (i.e. rs6267 [Ala22Ser] [28], rs740602, rs13306281 and rs3218737) was obtained from 150 subjects from the same population by means of sequence analysis using the ABI Prism 3130XL automated sequencer according to the instructions of the manufacturer (Applied Biosystems, The Netherlands). However, three of them appeared non-polymorphic, while rs740602 was found only once in the heterozygous state (minor allele frequency of $<0.01$ ) and all were excluded from further analysis. For the case-control study, genotype data (rs4680) from 169 cases with a history of venous thrombosis was obtained.

\section{Statistical analyses}

Haploview (29) was used to evaluate linkage disequilibrium (LD) and correlations between the SNPs. Haplotype association analyses for homocysteine levels were performed using Whap. Whap (http://pngu.mgh.harvard.edu/purcell//whap) takes the ambiguity in individual haplotype estimations into account by applying a weighted likelihood approach. Prior to the haplotype analyses, the log transformed Hcy values were standardized. We used permutation testing as implemented in the Whap program to correct for multiple testing in all analyses. Haplotypes with a frequency of less than $2 \%$ were excluded from the analysis. Singlelocus genotype effects on (log-transformed) metabolites were evaluated by linear regression analysis (SPSS 12.0). In the haplo- 
Table 3: Linkage disequilibrium coefficient (D') between SNPs across the COMT gene in controls. Above the diagonal the D'-values are shown and below the diagonal their corresponding squared correlation coefficients (in italics).

\begin{tabular}{|l|l|l|l|l|l|l|}
\hline Variant & Rs number & $\begin{array}{l}\text { Distance from } \\
\text { rs2097603 (bp) }\end{array}$ & rs2097603 & rs4633 & rs4680 & rs174699 \\
\hline 1 & rs2097603 & 0 & - & 0.427 & 0.438 & 0.50 \\
\hline 2 & rs4633 & 22143 & 0.149 & - & 0.964 & 0.627 \\
\hline 3 & rs4680 & 23179 & 0.165 & 0.89 & - & 0.695 \\
\hline 4 & rs 174699 & 26366 & 0.016 & 0.033 & 0.038 & - \\
\hline
\end{tabular}

type analysis, changes in homocysteine levels are expressed relative to the most frequent haplotype group. Odds ratios (ORs) were estimated using logistic regression analysis (SPSS 12.0).

\section{Results}

\section{Population charateristics and genotyping}

Baseline characteristics of the study populations are shown in Table 1.

For details about these SNPs regarding location, function and validation method, see Table 2. The four COMT SNPs under study were all in Hardy-Weinberg equilibrium ( $p>0.4$ ). Minor allele frequencies for rs2097603, rs4633, rs4680 and rs174699 were 0.48 (G allele), 0.47 (C allele), 0.49 (G allele) and 0.068 (C allele), respectively. Of note, the $324 \mathrm{G}$ allele (which is presumed to be the wild-type allele of rs4680) had a lower frequency than the 324A allele, which is also observed in other studies (Table 2). Linkage disequilibrium (D') and squared correlation coefficients between each of the genotyped variants are shown in Table 3. The SNP pair rs4680 (324G $>A)$ and rs4633 (36T $>C)$ was in almost complete LD, with a D' of 0.96 and a squared correlation $\left(\mathrm{r}^{2}\right)$ of 0.89 . No strong LD was observed for the other SNP pairs.

\section{Haplotype associations in the general population}

Genotype data for all four polymorphisms was obtained from 401 individuals (mean age $50.6 \pm 13.3$ years), from which $41.0 \%$ was male $(\mathrm{n}=164)$. Mean fasting tHcy was $10.4(95 \%$ CI 10.0 to 10.8) $\mu \mathrm{M}$, serum creatinin was $74.3(95 \%$ CI 72.7 to 75.9$) \mu \mathrm{M}$, vitamin B12 was 221 (95\% CI 208 to 234) pM and folate was $13.1(95 \%$ CI 12.3 to 13.9$) \mathrm{nM}$. tHcy was correlated with plasma folate, red blood cell folate and vitamin B12 (Spearmann $\rho$ values were $-0.21,-0.24$ and -0.31 , respectively, with $p<0.01$ at a two-tailed level).

The haplotype frequencies and their relative effects on tHcy are presented in Table 4. By omitting the haplotypes with a frequency of less than two percent, $95 \%$ of the haplotypes was covered. The omnibus association test using all haplotypes for crude fasting thcy showed a bordeline significant effect $(\mathrm{p}=0.05)$. Haplotype specific analysis, i.e. analysis of the effect of the haplotype relative to the most common haplotype, showed that the effect was mainly due to the G-C-G-T haplotype that was statistically significant associated with low tHcy levels (-13.3\% [95\% CI -23.6 to -3.1$]$, $\mathrm{p}=0.01$ ). Adjustment for age, sex, serum creatinine, MTHFR $677 \mathrm{C}>\mathrm{T}$ polymorphism and plasma folate did not change this point estimate (not shown). The omnibus haplotype test was no longer significant when the analysis was conducted conditional on rs 4680 or on rs 4633 ( $p=0.28$ and $p=0.26$, respectively). This means that the haplotype analysis did not reveal major additional effects besides that observed for the single-loci. Indeed, an increase of $10.4 \%$ ( $95 \% \mathrm{CI} 0.01$ to $0.21, \mathrm{p}=0.03)$ and $8.8 \%(95 \% \mathrm{CI}-0.00$ to 0.18 , $\mathrm{p}=0.06$ ) in tHcy was observed for COMT 324AA and 324GA (rs 4680) individuals, respectively, when compared with 324GG subjects (Table 5). None of the genotypes was associated with folate, red blood cell folate or vitamin B12 levels (not shown). Moreover, the fact that adjustment for folate status (an important determinant of tHcy) did not influence the association between the COMT haplotype and tHcy suggests that the observed genotype-phenotype association is not confounded by differences in vitamin status.

Furthermore, by comparing a model in which the effects of the three haplotypes containing allele $\mathrm{C}$ and $\mathrm{G}$ at the second and third locus were constrained to be equal to a model in which all haplotype effects were estimated separately, we found that the effects of the N-C-G-N haplotypes (where $\mathrm{N}$ represents one of the alleles observed at the first and fourth locus) were not different $(\mathrm{p}=0.18)$. This suggests that the haplotype background on which these variants appear had no major influence on tHcy.

\section{The COMT 324G>A variant and recurrent venous thrombosis risk}

Genotype data of the COMT 324G>A (rs4680) polymorphism was obtained from 424 controls and 169 , from which $41 \%$ $(\mathrm{n}=174)$ and $51 \%(\mathrm{n}=86)$ were male, respectively. For the controls, mean age and blood parameters were similar as those individuals included in the haplotype analysis. For the recurrent venous thrombosis cases (mean age $61.5 \pm 14.3$ years) geometric mean $(95 \% \mathrm{CI})$ tHcy was $12.6(11.6$ to 13.6$) \mu \mathrm{M}, 83.1$ ( 78.6 to 87.6) $\mu \mathrm{M}$ for serum creatinin, 237 (207 to 267 ) $\mathrm{pM}$ for vitamin $\mathrm{B} 12$ and 13.7 (12.4 to 15.0$) \mathrm{nM}$ for folate.

The genotype frequencies were $34.9 \%(\mathrm{n}=59), 45.6 \%$ $(\mathrm{n}=77), 19.5 \%(\mathrm{n}=33)$, and $27.6 \%(\mathrm{n}=117), 47.6 \%(\mathrm{n}=202)$, $24.8 \%(\mathrm{n}=105)$ for AA, AG, GG genotypes in cases and controls,

Table 4: Haplotype frequencies and relative change in tHcy in the general population using the G-T-A-T haplotype (corresponding with SNP I, 2, 3 and 4, respectively) as a reference.

\begin{tabular}{|l|l|l|}
\hline Haplotype & Frequency & Crude change tHcy \% $[95 \%$ CI $]$ \\
\hline G-T-A-T & 0.355 & $0^{\mathrm{a}}$ \\
\hline A-C-G-T & 0.312 & $-3.2[-9.7$ to 3.4$]$ \\
\hline A-T-A-T & 0.167 & $1.3[-7.3$ to 9.8$]$ \\
\hline G-C-G-T & 0.120 & $-13.3[-23.6 \text { to }-3.1]^{\mathrm{b}}$ \\
\hline A-C-G-C & 0.046 & $-3.3[-15.8$ to 9.2$]$ \\
\hline${ }^{2}$ Reference category, ${ }^{\text {p }}$-ANOVA $=0.01$. \\
\hline
\end{tabular}


Table 5: COMT single-locus genotype effects on tHcy concentrations in population-based controls.

\begin{tabular}{|c|c|c|c|c|c|c|}
\hline Variant & Genotype & n (\%) & $\mathrm{MAF}^{\mathrm{b}}$ & $\begin{array}{l}\text { Crude mean tHcy } \\
{[95 \% \mathrm{Cl}](\mu \mathrm{M})}\end{array}$ & $\begin{array}{l}\text { Crude change \% } \\
{[95 \% \mathrm{Cl}]}\end{array}$ & P-value \\
\hline rs2097603 & AA & $117(27.7)$ & & 10.7 [10.0 to 11.5$]$ & $0^{a}$ & \\
\hline \multirow[t]{2}{*}{$(n=422)$} & AG & $208(49.3)$ & & $10.3[9.8$ to 10.8$]$ & $-4.1[-12.1$ to 4.7$]$ & \\
\hline & GG & $97(23.0)$ & $0.48(G)$ & $10.3[9.5$ to II.I] & $-4.2[-13.6$ to 6.3$]$ & 0.474 \\
\hline rs4633 & $\mathrm{TT}$ & $120(29.1)$ & & $10.8[10.4$ to $|1| .5]$ & $0^{a}$ & \\
\hline \multirow[t]{2}{*}{$(n=4 \mid 3)$} & TC & $199(48.2)$ & & 10.4 [9.9 to |l 1.0$]$ & $-3.0[-11.0$ to 5.6$]$ & \\
\hline & $\mathrm{CC}$ & $94(22.8)$ & $0.47(C)$ & $10.0[9.2$ to 10.8$]$ & $-7.3[-16.3$ to 2.6$]$ & 0.143 \\
\hline rs4680 & GG & $105(24.8)$ & $0.49(\mathrm{G})$ & $9.6[8.7$ to 10.7$]$ & $0^{\mathrm{a}}$ & \\
\hline \multirow[t]{2}{*}{$(\mathrm{n}=424)$} & GA & $202(47.6)$ & & I0.5 [9.6 to II.5] & $8.8[-0.00$ to 0.18$]$ & \\
\hline & $\mathrm{AA}$ & $117(27.6)$ & & $10.8[10.0$ to $\mid 1.5]$ & $10.4[0.01$ to 0.21$]$ & 0.036 \\
\hline rs174699 & $\mathrm{TT}$ & $365(86.9)$ & & $10.4[10.0$ to 10.8$]$ & $0^{\mathrm{a}}$ & \\
\hline \multirow[t]{2}{*}{$(n=420)$} & TC & $53(12.6)$ & & $10.2[9.2$ to $\mid 1.3]$ & $-2.4[-12.7$ to 9.1$]$ & \\
\hline & $\mathrm{CC}$ & $2(0.5)$ & $0.07(\mathrm{C})$ & $10.7[6.3$ to 18.3$]$ & $2.8[-39.8$ to 75.1$]$ & 0.748 \\
\hline
\end{tabular}

respectively. These correspond to minor $(\mathrm{G})$ allele frequencies of 0.42 and 0.49 for cases and controls, respectively. We found that individuals with the 324AA genotype, corresponding with those having the highest tHcy, were at higher risk for recurrent venous thrombosis compared with $324 \mathrm{GG}$ subjects, although this estimation did not reach statistical significance (OR 1.61 [95\%CI 0.97 to 2.65], $\mathrm{p}=0.06$ ). The OR for individuals with the 324GA genotype was $1.21(95 \% \mathrm{CI} 0.76$ to $1.94, \mathrm{p}=0.18)$. The other genetic variants were not associated with recurrent venous thrombosis risk (not shown)

\section{Discussion}

We screened for the rs 4680 variant as well as three other SNPs dispersed over the COMT gene (rs2097603, rs4633 and rs 174699) and performed haplotype analysis in order to identify whether a specific haplotype was related to tHcy. We show that rs4680 was singularly associated with tHcy levels in a Dutch population study, and was responsible for the observed haplotype effect. The small, but non-significant, effect on tHcy that was observed for variant rs4633 (not shown) is likely to be explained by its high correlation with rs 4680 (Table 2$)(20,30)$. Interestingly, the 324AA genotype (rs4680) was more prevalent in venous thrombosis cases suggesting that this variant may affect recurrent venous thrombosis risk as well.

The $324 \mathrm{G}>\mathrm{A}$ ( $\mathrm{rs} 4680$ ) polymorphism has been extensively studied for its effect at the molecular level, mostly because of its potential role in schizophrenia susceptibility. Functional studies showed that the COMT 324AA genotype is associated with decreased enzyme activity in vitro and in human brain extracts (22, 31) although the Val-allele was expressed at a slightly lower level in human brain (23). In the past, Goodman et al. (19) showed that the COMT $324 \mathrm{G}>\mathrm{A}$ variant affected tHcy in controls, while Geisel et al. did not find such an effect in elderly subjects (32). In addition, it has been suggested that other variants might explain the observed associations (20). Our results show that the 324AA ge- notype is significantly associated with increased tHcy levels, which may support the observation of higher expression of the Met-allele by Bray et al. (23). The higher levels of tHcy associated with the 324AA genotype may explain why these subjects tend to have a higher risk for venous thrombosis. However, a disturbed methylation by COMT in itself may also be involved, especially since the vascular system is constantly exposed to circulating catecholamines and catecholestrogens. It has been shown that catecholestrogens may have beneficial effects on the vascular system by reducing fibrinogen and overall fibrinolysis potential (33), although negative effects have also been reported (16). The measurement of plasma AdoMet and AdoHcy levels, the ratio of which is a marker of methylation capacity, and in-vitro methylation studies could provide additional evidence for disturbed methylation in subjects carrying this variant. It should be noted that we included patients with a history of venous thrombosis, which may give an overestimation of the relative risk. Additional studies are required to study whether the COMT $324 \mathrm{G}>\mathrm{A}$ polymorphism is related to a first thrombotic event.

One may raise the question whether it is plausible that the flux through the COMT enzyme is high enough to generate a relatively large difference in tHcy (about $10 \%$ ) between subjects having the 324GG and 324AA genotype. Studies with Parkinson's disease patients whose tHcy levels rose upon L-DOPA treatment (34-36), indicate that a higher COMT flux is reflected in plasma tHcy levels. In addition, a recent genome-wide linkage scan performed by Souto et al. identified another methyltransferase, Nicotinamide N-methyltransferase (NNMT), as a possible major determinant of tHcy (37). This shows that not only methyltransferases with a high flux-rate, like guanidinoacetate- and phosphatidylethanolamine methyltransferase (15), contribute to homocysteine synthesis, but also methyltransferases with an apparently modest contribution to overall methyltransferase activity. In conclusion, the 324AA genotype (rs4680) is associated with increased tHcy in the general population. Subjects with the 324AA genotype also tend to have a higher risk for recurrent ve- 
nous thrombosis compared to subjects with the $324 \mathrm{GG}$ genotype. These data may give a hint as to what is the high-risk allele in COMT-related disorders, like cardiovascular disease and schizophrenia in particular.

\section{Acknowledgments}

This study was in part supported by grant 2002B68 from the Netherlands Heart Foundation. Martin den Heijer, MD, PhD, is supported by a VENIgrant from the Dutch Organization for Scientific Research (NWO).

\section{References}

1. The Homocysteine Studies Collaboration. Homocysteine and risk of ischemic heart disease and stroke: a meta-analysis. JAm Med Assoc 2002; 288: 2015-2022. 2. Wald DS, Law M, Morris JK. Homocysteine and cardiovascular disease: evidence on causality from a meta-analysis. Br Med J 2002; 325: 1202.

3. den Heijer M, Lewington S, Clarke R. Homocysteine, MTHFR and risk of venous thrombosis: a metaanalysis of published epidemiological studies. J Thromb Haemost 2005; 3: 292-299.

4. Clarke R, Smith AD, Jobst KA, et al. Folate, vitamin B12, and serum total homocysteine levels in confirmed Alzheimer disease. Arch Neurol 1998; 55: 1449-1455.

5. Czeizel AE. Primary prevention of neural-tube defects and some other major congenital abnormalities: recommendations for the appropriate use of folic acid during pregnancy. Paediatr Drugs 2000; 2: 437-449.

6. Muntjewerff JW, Kahn RS, Blom HJ, et al. Homocysteine, methylenetetrahydrofolate reductase and risk of schizophrenia: a meta-analysis. Mol Psychiatry 2006; 11: 143-149.

7. Undas A, Brozek J, EzczeklikA. Homocysteine and thrombosis: from basic science to clinical evidence. Thromb Haemost 2005; 94: 907-915.

8. James SJ, Melnyk S, Pogribna M, et al. Elevation in S-adenosylhomocysteine and DNA hypomethylation: potential epigenetic mechanism for homocysteine-related pathology. J Nutr 2002; 132 (8 Suppl): 2361S-2366S

9. Abdolmaleky HM, Cheng KH, Russo A, et al. Hypermethylation of the reelin (RELN) promoter in the brain of schizophrenic patients: a preliminary report. Am J Med Genet B Neuropsychiatr Genet 2005; 134 : 60-66.

10. Bjorklund NK, Gordon R. A hypothesis linking low folate intake to neural tube defects due to failure of post-translation methylations of the cytoskeleton. Int J Dev Biol 2006; 50: 135-141.

11. Keijzer MB, den Heijer M, Borm GF, et al. Low fasting methionine concentration as a novel risk factor for recurrent venous thrombosis. Thromb Haemost 2006; 96: 492-497.

12. Yi $P$, Melnyk $S$, Pogribna $M$, et al. Increase in plasma homocysteine associated with parallel increases in plasma S-adenosylhomocysteine and lymphocyte DNA hypomethylation. J Biol Chem 2000; 275: 29318-29323.
13. Castro R, Rivera I, Struys EA, et al. Increased homocysteine and S-adenosylhomocysteine concentrations and DNA hypomethylation in vascular disease. Clin Chem 2003; 49: 1292-1296.

14. Mattson MP. Methylation and acetylation in nervous system development and neurodegenerative disorders. Ageing Res Rev 2003; 2: 329-342.

15. Brosnan JT, Jacobs RL, Stead LM, et al. Methylation demand: a key determinant of homocysteine metabolism. Acta Biochim Pol 2004; 51: 405-413.

16. Zhu BT. On the mechanism of homocysteine pathophysiology and pathogenesis: a unifying hypothesis. Histol Histopathol 2002; 17: 1283-1291.

17. Zhu BT. CNS dopamine oxidation and catecholO-methyltransferase: importance in the etiology, pharmacotherapy, and dietary prevention of Parkinson's disease. Int J Mol Med 2004; 13: 343-353.

18. Clarke, Banfield. Chapter 7: S-adenosylmethionine-dependent methyltransferases. Homocysteine in health and disease. Cambridge University Press, 2001.

19. Goodman JE, Lavigne JA, Wu K, et al. COMT genotype, micronutrients in the folate metabolic pathway and breast cancer risk. Carcinogenesis 2001; 22: 1661-1665.

20. Shifman S, Bronstein M, Sternfeld M, et al. A highly significant association between a COMT haplotype and schizophrenia. Am J Hum Genet 2002; 71: 1296-1302.

21. Zhu BT. Medical hypothesis: Hyperhomocysteinemia is a risk factor for estrogen-induced hormonal cancer. Int J Oncol 2003; 22: 499-508.

22. Chen J, Lipska BK, Halim N, et al. Functiona analysis of genetic variation in catechol-O-methyltransferase (COMT): effects on mRNA, protein, and enzyme activity in postmortem human brain. Am J Hum Genet 2004; 75: 807-821.

23. Bray NJ, Buckland PR, Williams NM, et al. A haplotype implicated in schizophrenia susceptibility is associated with reduced COMT expression in human brain. Am J Hum Genet 2003; 73: 152-161

24. den Heijer M, Blom HJ, Gerrits WB, et al. Is hyperhomocysteinaemia a risk factor for recurrent venous thrombosis? Lancet 1995; 345: 882-885.

25. Muntjewerff JW, Gellekink H, den Heijer M, et al. Polymorphisms in catechol-O-methyltransferase and methylenetetrahydrofolate reductase in relation to the risk of schizophrenia. Eur Neuropsychopharmacol 2007; epub ahead of print.
26. te Poele-Pothoff MT, van den Berg M, Franken DG, et al. Three different methods for the determination of total homocysteine in plasma. Ann Clin Biochem 1995; 32: $218-220$.

27. Miller SA, Dykes DD, Polesky HF. A simple salting out procedure for extracting DNA from human nucleated cells. Nucleic Acids Res 1988; 16: 1215.

28. Lee SG, Joo Y, Kim B, Chung S, et al. Association of Ala72Ser polymorphism with COMT enzyme activity and the risk of schizophrenia in Koreans. Hum Genet 2005; 116: 319-328.

29. Barrett JC, Fry B, Maller J, et al. Haploview: analysis and visualization of LD and haplotype maps. Bioinformatics 2005; $21: 263-265$.

30. Handoko HY, Nyholt DR, Hayward NK, et al. Separate and interacting effects within the catecholO-methyltransferase (COMT) are associated with schizophrenia. Mol Psychiatry 2005; 10: 589-597.

31. Lotta T, Vidgren J, Tilgmann C, et al. Kinetics of human soluble and membrane-bound catechol O-methyltransferase: a revised mechanism and description of the thermolabile variant of the enzyme. Biochemistry 1995; 34: 4202-4210.

32. Geisel J, Hubner U, Bodis M, et al. The role of genetic factors in the development of hyperhomocysteinemia. Clin Chem Lab Med 2003; 41: 1427-1434.

33. Mendelsohn ME, Karas RH. The protective effects of estrogen on the cardiovascular system. N Engl J Med 1999; 340: 1801-1811.

34. Lamberti P, Zoccolella S, Armenise E, et al. Hyperhomocysteinemia in L-dopa treated Parkinson's disease patients: effect of cobalamin and folate administration. Eur J Neurol 2005; 12: 365-368.

35. Lamberti P, Zoccolella S, Iliceto G, et al. Effects of levodopa and COMT inhibitors on plasma homocysteine in Parkinson's disease patients. Mov Disord 2005; 20: 69-72.

36. Yasui K, Nakaso K, Kowa H, et al. Levodopa-induced hyperhomocysteinaemia in Parkinson's disease. Acta Neurol Scand 2003; 108: 66-67.

37. Souto JC, Blanco-Vaca F, Soria JM, et al. A genomewide exploration suggests a new candidate gene at chromosome 11q23 as the major determinant of plasma homocysteine levels: results from the GAIT project. Am J Hum Genet 2005; 76: 925-933. 